\title{
Macrosomia in developing countries
}

Associations between neonatal macrosomia and its risk factors in 23 developing countries in Africa, Asia and Latin America mirror those reported for developed countries, a large international study published in The Lancet has shown.

Obesity and diabetes mellitus are wellknown risk factors for macrosomia, which in turn increases the risk of obstructed labour. "I had seen many tragic cases of uterine rupture, maternal death, and stillbirths due to obstructed labour in Niger during 2 years working there as a paediatrician and gynaecologist," recounts lead author Ai Koyanagi (Hatsuishi Hospital, Kashiwa, Japan). Koyanagi and colleagues set out to investigate whether excessive maternal and perinatal morbidity and mortality is associated with macrosomia in developing countries, for which only limited data exist.

The researchers analysed data from 276,436 births recorded via the WHO Global Survey on Maternal and Perinatal Health and assessed risk factors for macrosomia and caesarean section, and adverse maternal and perinatal outcomes.
Using the $90^{\text {th }}$ percentile of countryspecific birthweight values as the cut-off value, Koyanagi and co-workers found that diabetes mellitus and obesity were strong risk factors for macrosomia in all regions. In turn, macrosomia was associated with increased risks of caesarean section and adverse maternal birth outcomes in all regions. An association between macrosomia and adverse perinatal outcomes was seen only in Africa.

"Although screening and management of diabetes mellitus during pregnancy may not be considered a priority in many developing countries, our study highlights the potential importance of screening for and managing this condition during pregnancy to avoid macrosomia and its associated adverse pregnancy outcomes," concludes Koyanagi.

Linda Koch

Original article Koyanagi, A. et al. Macrosomia in 23 developing countries: an analysis of a multicountry, facility-based, cross-sectional survey. Lancet doi:10.1016/ S0140-6736(12)61605-5 